\title{
ADICIONES A DARWIN'S INSECTS SMITH (1987): CHINCHES (HEMIPTERA: HETEROPTERA) DE CHILE ${ }^{1}$
}

\author{
ADDITIONS TO DARWIN'S INSECTS SMITH (1987): BUGS \\ (HEMIPTERA:HETEROPTERA) FROM CHILE
}

Vicente Pérez ${ }^{2}$

Smith (1987) dio a conocer la lista de las especies de insectos recolectados por Charles Darwin en la expedición inglesa (1831-1836) del H.M.S. Beagle, aunque advierte que aún puede haber otras especies ya que el material recogido por Darwin no tiene un solo repositorio.

Esta nota tiene por objeto adicionar tres especies de chinches no registradas por Smith (1987), recolectadas por Darwin, pertenecientes a la colección del British Museum (Natural History) y consignadas como material adicional por Scudder (1962) y China (1963) al publicar los resultados de la expedición de la Royal Society (1958-1959) a Chile austral.

Familia Lygaeidae (Schiller, 1829)

Bergidia polychroma (Spinola, 1852).

Pachymerus polychromus Spinola, 1852.

In Gay, C., Historia Física y Politica de Chile, Zool. 7:179. Scudder (1962): "Chile, Chiloé".

Familia Pentatomidae (Leach, 1815)

Subfamilia Acanthosomatinae Stal, 1864

Ditomotarsus gayi Spinola, 1852. In Gay, C., Historia Física y Política de Chile, Zool. 7:127, pl. 1, fig.8. China (1963):

"There are two specimens in the British Museum (Nat. Hist.) collected on Chiloe Is. by Charles Darwin, Voyage of Beagle".
Familia Aradidae (Spinola, 1837)

Subfamilia Isoderminae Stal, 1872

Isodermus gayi (Spinola, 1852)

Anchomichon gayi Spinola, 1852. In Gay,

C., Historia Física y Política de Chile, Zool. $7: 214$.

Ecpiestocoris castaneus Blanchard, 1852. In Gay, C., Historia Física y Politica de Chile, Zool. 7:223. China (1963): "There is a series of ten specimens in the British Museum (Nat. Hist.) collected by Charles Darwin on the Hardy Peninsula [5530' S; 68¹5' W], Cape Horn, during the voyage of the Beagle". "Es errónea la cita "Cape Horn".

En Smith (1987) no están registrados Scudder (1962) ni China (1963) en Referencias Bibliográficas; y las tres especies de chinches que se agregan en esta nota tampoco están consignadas en el Índice Científico.

En la sección Insect Notes, que constituye la parte medular de Darwin's Insects, ordenadas por año de recolección y entradas numerales, la localidad Chiloé presenta numerosas entradas correspondientes a los años 1834 y 1835 con algunos registros de Hemiptera, pero sin ninguna del suborden Heteroptera.

La localidad península Hardy, situada en la

${ }^{1}$ Proyecto Interno Estudio de Biodiversidad, Distribución y Relaciones Biogeográficas de la Entomofauna en Fuego-Patagonia

${ }^{2}$ Laboratorio de Entomología. Instituto de la Patagonia, Universidad de Magallanes. Casilla 113-D. Punta Arenas, Chile. vicente.perez@umag.cl 
isla Hoste, está registrada dos veces: en la figura 4 ("Tierra del Fuego, que muestra las localidades mencionadas en las Insect Notes y otros ítems de interés de Darwin") y en una entrada: "[año] 1833... Tierra del Fuego...[entrada] 1005...Hardy Peninsula", pero como localidad de recolección de coleópteros y no de hemípteros.

\section{LITERATURA CITADA}

China, W.E. 1963 (1962). Hemiptera-Heteroptera collected by the Royal Society Expedition to South Chile 1958-1959. Ann. Mag. Nat. Hist.(13) 5:706-723.

Scudder, G.G.E. 1962. Results of the Royal Society Expedition to Southern Chile.1958-59: Lygaeidae (Hemiptera), with the Description of a New Subfamily. The Canadian Entomologist 94:1064-1075.

Smith, K.G.V (Editor). 1987. Darwin's Insects. Charles Darwin's Entomological Notes. Bulletin of the British Museum (Natural History). Historical 